\title{
Collaborative Requirements Elicitation with Visualization Techniques
}

\author{
Diogo Duarte \\ Instituto Superior Técnico \\ Lisbon, Portugal \\ diogo.m.duarte@ist.utl.pt
}

\author{
Carla Farinha \\ Instituto Superior Técnico \\ Lisbon, Portugal \\ carla.farinha@opensoft.pt
}

\author{
Miguel Mira da Silva \\ Instituto Superior Técnico \\ Lisbon, Portugal \\ mms@ist.utl.pt
}

\author{
Alberto Rodrigues da Silva \\ Instituto Superior Técnico \\ Lisbon, Portugal \\ alberto.silva@acm.org
}

\begin{abstract}
Requirements elicitation is one of the first activities that tries to define the project scope and elicit user requirements. This activity relies in communication and cooperation between stakeholders which makes collaboration crucial for the success of this activity, especially in global software development projects with distributed teams and stakeholders. Despite the need for collaboration, lack of user input is still one of the problems of requirements elicitation, having negative consequences on project success. In this paper we present a proposal to involve stakeholders during requirements elicitation through the support of online collaboration and the usage of visualization techniques to stimulate stakeholders and increase their awareness about requirements. We are following action research methodology and present the results of a recent experiment.
\end{abstract}

Keywords-online collaboration, requirements elicitation, user involvement, requirements visualization, action research.

\section{INTRODUCTION}

Software projects are shaped by requirements which can be grouped in distinct categories, namely business, user, functional or non-functional requirements. Requirements Engineering (RE) deals with requirements development and management [1]. One of the first activities of requirements development is requirements elicitation.

Requirements elicitation is a critical activity in RE. This activity's goal is to understand the stakeholders' needs and constraints, which will be analyzed and specified with requirements [1]. User requirements include tasks that the users need the system to support and their expectations about performance and other quality attributes.

For the success of this activity communication and collaboration between the stakeholders is essential, since communication problems and conflicts between stakeholders that occur [2]. The worldwide distribution of teams and stakeholders reinforce the need for collaboration and communication support in RE [3] [4].

Despite the need for collaboration, lack of user involvement continues to be a problem related to requirements elicitation [1] [5] [6] [7]. This problem can lead to requirements that are identified in later phases of development, delaying the project and demanding the need of code rewriting [1] [5]. Another reason for involving users is that sometimes customers are more worried with financial facts while users are experts in their domain and know what tasks need to be supported by the system [6].
Benefits of user involvement in requirements elicitation have been pointed out, such as higher requirements quality, documentation of essential customer and user needs, political conflict reduction and higher acceptance of the system [6] [8] [9].

In our research we intend to involve users in requirements elicitation through an online collaborative approach. In order to increase the stakeholders' perception of requirements and their motivation to be involved in the elicitation activity, we propose the usage of visualization techniques. These techniques are proposed because at each project we will be creating an online community to elicit requirements and a problem of online communities is lack of participation [10], which combined with lack of user involvement in requirements elicitation justifies the need to motivate the stakeholders for this activity. Difficulties to motivate the stakeholders for requirements elicitation have also been pointed out by Kujala [8].

The methodology followed in this research is Action research, which is composed by five phases: diagnosing, action planning, action taking, evaluating and specifying learning [11]. We have completed an entire cycle of research according to this methodology, the problem of lack of user involvement in requirements elicitation has been identified, a proposal has been designed and implemented and conclusions have been reached.

The remaining of the paper is as follows. Section II introduces the related work of collaborative requirements elicitation, requirements visualization and participation in online communities. Section III introduces our proposal for elicitation based on collaboration and visualization techniques. The prototype that has been built to do the evaluation of our proposal is described in Section IV. Our experiment and its results are presented in Sections V and VI. Evaluation is made in VII. Conclusion and future work description is described in Section VIII.

\section{RELATED WORK}

This section introduces collaborative requirements elicitation approaches and different approaches for requirements visualization. Since we propose to elicit requirements with an online collaborative platform we also introduce participation in online communities, namely related to the use of social 
visualization, which is part of our proposal and has been used as a way to increase user participation.

We argue that some of the current approaches do not explore requirements visualization as a mean to increase the awareness and understanding around requirements. Additionally, approaches to increase user involvement like social visualization are not used.

\section{A. Collaborative Requirements Elicitation}

Group work is one way to collaboratively elicit requirements through the promotion of stakeholders' cooperation and commitment [2]. Group meetings for requirements elicitation include Brainstorm, JAD or Focus Group [2]. For example, Farinha and Mira da Silva have used Focus Group both in face-to-face and online discussions [12] [13].

A problem associated with group meetings is the difficulty to organize and schedule a meeting due to the number of stakeholders that may be involved in the project [2]. Davidson has also pointed out JAD as a time consuming activity and only adequate for small scale projects [14].

Alternative models to group meetings have been proposed, like WinWin [15] and EasyWinWin [16], CoREA [17] and Athena [18]. The last two consider a distributed environment for collaboration supported by electronic tools.

In addition, the use of wikis has been proposed to deal with distributed teams facilitating and increasing the participation of all project stakeholders [19] [20].

A wiki is collaborative software that allows users to add, remove, and amend content on a common platform, which can be a public web site or a more constrained company intranet [21]. Some of the common features of wikis are centralized and shared communication, possibility to view previous versions of a document, watch-listing, searchability and categorization [21].

Some proposals to provide a collaborative environment for elicitation based on wikis have been made by [22] [23] [24] [25].

For example Softwiki supports distributed requirements submission and discussion, requirements classification and prioritization and requirements visualization techniques such as: [22]

1) Graph with connections between related requirements

2) Tag cloud visualization for the mentioned terms

3) Geographical map with the origin of the requirements

ShyWiki [23] makes a distributed brainstorming session possible. Requirements can be prioritized through voting. WikiWinWin [24] is a requirements negotiation tool that supports EasyWinWin methodology. SmartWiki [25] provides requirements definition through document templates, also offering feedback on the specified requirements through heuristics. Support for glossary and project management activities is also provided.

A disadvantage pointed out to wikis is conflicts among users which can also lead to misunderstandings around requirements that may arise from the stakeholders' different backgrounds and objectives [19].
However, online elicitation tools are not restricted to wikis, other proposals for requirements elicitation have been made [26] [27] [28].

Stakesource 2.0 uses social networks and collaborative filtering to suggest requirements that the stakeholder may prefer [26]. Stakeholders can invite other stakeholders to participate and afterwards they suggest and rate requirements.

Annotation Tool [27] is an evolution of Annotate!Pro that lets the user takes screenshots and annotate them with their proposals for project improvement.

iRequire [28] is a tool for mobile phones and enables users to blog their requirements whenever their need is triggered. The main features of iRequire are the possibility to take a picture of the environment, document a user need, describe the main task and provide a rationale, and check the summary of a need.

These studies continue to address the problem of lack of stakeholder involvement in requirements elicitation.

\section{B. Visualization of Requirements}

Browsing a disjoint list of textual requirements may impede users to better understand requirements. Visualization can be a mean to achieve better understanding of the identified requirements [29].

Data might be represented through the use of visualization. This representation increases the awareness of an individual about the data. Visualization is employed in many domains, for example latter phases of soft development, such as program call graphs and source code visualization, to increase overall program comprehension; or it is used to support testing and debugging tasks.

Current visualizations used in software engineering are mainly based in UML. However these UML-based models are rarely designed with the goal of helping stakeholders to see requirements and their properties in a clearer and more understandable way. Requirements are more than mere textual descriptions of what the system is supposed to achieve. Metadata can be associated to requirements (for example, attributes for author identification, cost, or priority), turning them into multi-dimensional clusters of metadata.

Requirements visualization is a current research subject, having the majority of proposals addressed at the analysis and specification of requirements engineering. In what concerns the elicitation activity, some proposals suggest the use of tabular visualizations, quantitative visualizations of risks by using charts, and modeling of requirements through business processes [30].

Usage of mind maps [31] to gather requirements has also been suggested. A mind map can group requirements in different categories, where color and images can likewise be added to the mind map.

Commercial RE tools also support requirements visualization, for example, GatherSpace [32] with graphical use case definition. Borland's Caliber Analyst [33] supports requirements definition through scenarios and storyboards for requirements validation. IBM's Requirements Composer [34] 
includes business process diagrams, use case models and UI sketching.

\section{Participation in Online Communities}

A problem in a large number of online communities is the lack of participation. Only a small part of the community tends to contribute which can lead to a failure in that community [10].

The main reasons to contribute in online communities have been summarized in extrinsic motivations like rewards or personal needs, intrinsic motivations like altruism or reputation and interpersonal reasons such as affiliation or liking the community [35].

Motivating members to contribute to online communities is a research area that had some studies on the psychology field [10], explored the sense of uniqueness and dissimilarity [36], and displayed the value of each contribution [37].

Online communities suffer from participation issues that can be related to a lack of motivation to contribute. Social visualization has also been used to stimulate user engagement in online communities, this kind of visualization is part of our proposal and is described next.

Competition is a form of social comparison that motivates users to participate in online communities [38]. Social comparison can take place if users are able to see the behavior of other users and their own. Visualization has been used to create awareness of what is going on in the community but it is not frequently used to stimulate social comparison.

Usually, people want to be recognized in a positive way and perform actions to gain social reputation, since the potential benefit compensates the required effort [38].

A social visualization provides information about the presence, activities and other data of a member's social involvement in a community [39]. Social visualization portrays social data that can be defined as traces related to some specific activity [40]. This kind of visualization can be used to increase awareness in a social environment.

Attempts to use social visualization to motivate users have shown good results in increasing the users' motivation to participate in the community [41] [42]. Some guidelines for social visualization construction have been proposed by Erickson [39], such as absence of customization and use of a third person point of view, meaning that individuals should see the same thing and view themselves as the other members of the community will.

\section{PROPOSAL}

In this paper we propose a collaborative environment for requirements elicitation with both requirements and social visualization support. Since our proposal includes the creation of a community to submit and discuss requirements we incorporate some of the patterns suggested to design social interfaces like commenting, votes, reputation and rankings [43].

With this proposal we can tackle the problem introduced by the distribution of stakeholders that can difficult the communication among them. Since requirements elicitation can benefit from user involvement we also intend to invite the users to participate in this activity and use visualization techniques to engage and stimulate them to this activity.

We intend to better support users in requirements elicitation and avoid possible schedule and geographical constraints that may influence personal meetings for requirements elicitation. Our proposal is distinct from the ones previously presented because of our focus in the community that will elicit the requirements and the included visualizations techniques.

The use of alternative requirements visualization is suggested to provide a better understanding of the requirements to the participants in the elicitation activity as stated by Gotel et al [29]. Metadata associated to requirements like date and time of submission, number of votes and comments will be used in the proposed visualizations. Social visualization is recommended due to its positive results in motivating users of online communities.

We recommend using a web platform for inputting textbased requirements suggestions. The proposed requirements can then be used in the subsequent activities of requirements development, namely analysis, specification and validation.

A requirement is mainly formed by a title, a description and the category in which it fits in. Additionally, the date and time of a submission should be kept. Users ought to have the option to submit their requirements anonymously or to be identified.

Moreover users should be able to comment on the suggested requirements in order to improve the discussion around them. Date and time of submission is collected as in requirements submission and the user may or may not assume the authorship of a comment. In order to express their preference about requirements, users ought to be able to vote on their favorite suggestions. This way, the requirements list will also be prioritized.

\section{A. Requirements Visualizations}

1) Project Dashboard: We recommend the use of graphic artifacts to visualize information about the proposed requirements. Each (requirements elicitation) project has a dashboard that quickly allows users to know about recent activities and the most popular requirements. To show these details a total of five bar charts will be used in this dashboard, namely:

- Requirements with more votes (top10)

- Requirements with more comments (top10)

- Users that submitted more requirements (top10)

- Requirements with more comments in the last 24 hours (top 10)

- Participation by date, regarding number of submitted requirements, comments and votes per day

The charts that show information about requirements are clickable, giving direct access to the selected requirement's details. The first bar of each chart is highlighted in a different color.

2) Requirements Visualization: Regarding requirements visualization, we propose three graphical alternatives. The first one is based on the hierarchy obtained by the use of categories to group requirements. Hence, we propose using a treemap to 
display these two hierarchy levels. A treemap can represent large hierarchical collections with emphasis on the relevance of each node through size or color [44].

In the first level of the treemap's hierarchy all the possible requirements categories will be displayed. In the second level, we propose to display all the requirements associated to the selected category. The size of each element can be used to represent information about the requirements. In the first level, the larger element (category) will be the one that has the requirements with more votes. In the second level (see Figure 1), the size of each element (requirement) in the treemap will represent the number of votes the represented requirement has. Different colors are used to provide a better distinction between each element of the treemap.

The second requirements visualization is based on a tag cloud [45]. This visualization is compact and through the use of size, attention is drawn towards the largest and presumably most important items. Tag clouds are becoming popular in web sites and blogs.

Using a 3D tag cloud enables users to see the most relevant requirements at the same time (see Figure 2). Every element of this tag cloud is clickable, meaning the details of a requirement are accessible through one click.

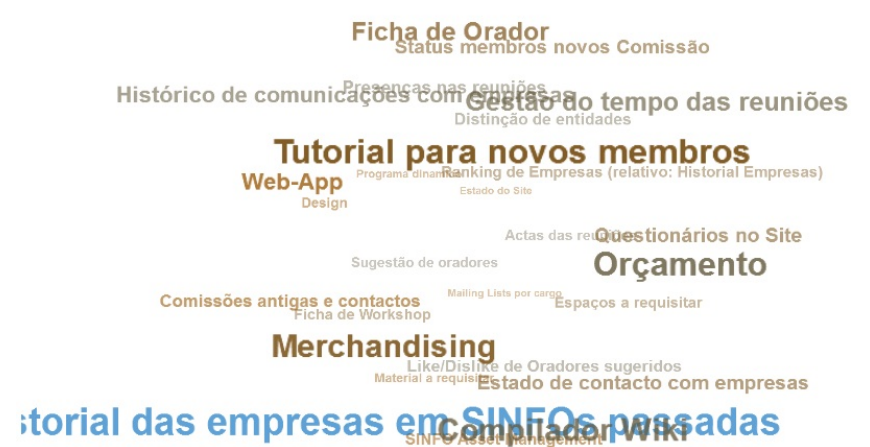

Fig. 2. 3D Tag cloud for requirements visualization

In addition, participants should be able to see the evolution of the number of comments and votes on requirements through time. This is possible through a bubble chart with a representation of time (motion chart): the number of votes and comments of each requirement will be used in the horizontal and vertical axis. Color will be employed to represent categories, meaning requirements from a given category will share the same color (see Figure 3).

Still, to give the participants a more traditional mode to visualize the proposed requirements a tabular requirements list is provided (with the title of each requirement, category, who submitted it and when as well as its number of votes ought to be available).

\section{B. Social Visualization}

Social visualization has a positive effect in motivating users of online communities to contribute to the given community, so we propose using social visualization as another mean to motivate users to participate in the elicitation activity.

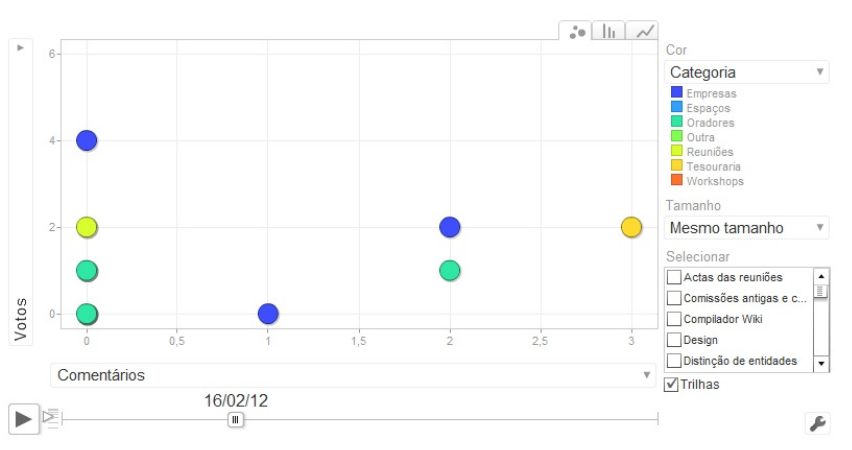

Fig. 3. Motion chart for requirements visualization through time

A bubble chart will be employed to illustrate the user's contributions (see Figure 4). The size of each bubble is intended to represent the total number of contributions (new requirements, comments and votes) that the same user has made, providing an effective way of detecting who is contributing more. Color will represent different user status that can be defined (like gold, silver and bronze) through the user's level of participation, adding a more competitive side to this visualization approach.

\section{PRototype}

To evaluate this proposal, a prototype has been built using the Outsystems agile platform [46]. This platform has been chosen due to its simplicity, short learning curve, and also for its capabilities of version control and easy deployment.

The developed prototype supports the features introduced in the previous section. The social visualization and requirements dashboard were implemented using Fusion Charts [47], a charting framework that is included in Outsystems agile platform. However, the proposed visualizations for the suggested requirements were built using Google Charts API [48] due to the lack of equivalent support by Fusion Charts.

\section{A. Requirements Submission, Discussion and Voting}

The prototype enables users to submit new requirements, discuss the existent ones through comments, and prioritize by using votes. Requirements submission and their comments can be signed by users with their username or be anonymous in order to avoid organizational barriers, which can intimidate the user when submitting an opinion.

A notification system based on comments has also been implemented in order to notify the users of recent activity. After each comment on requirements, the system sends an email to the author of the requirement and to the individuals that already posted a comment on the given requirement, informing that a new comment has been submitted.

At the end of the requirements elicitation process, a list of the suggested requirements can be exported to a xls file. This list contains the information related to each requirement including title, description, category, number of votes, author, date and time of submission. 


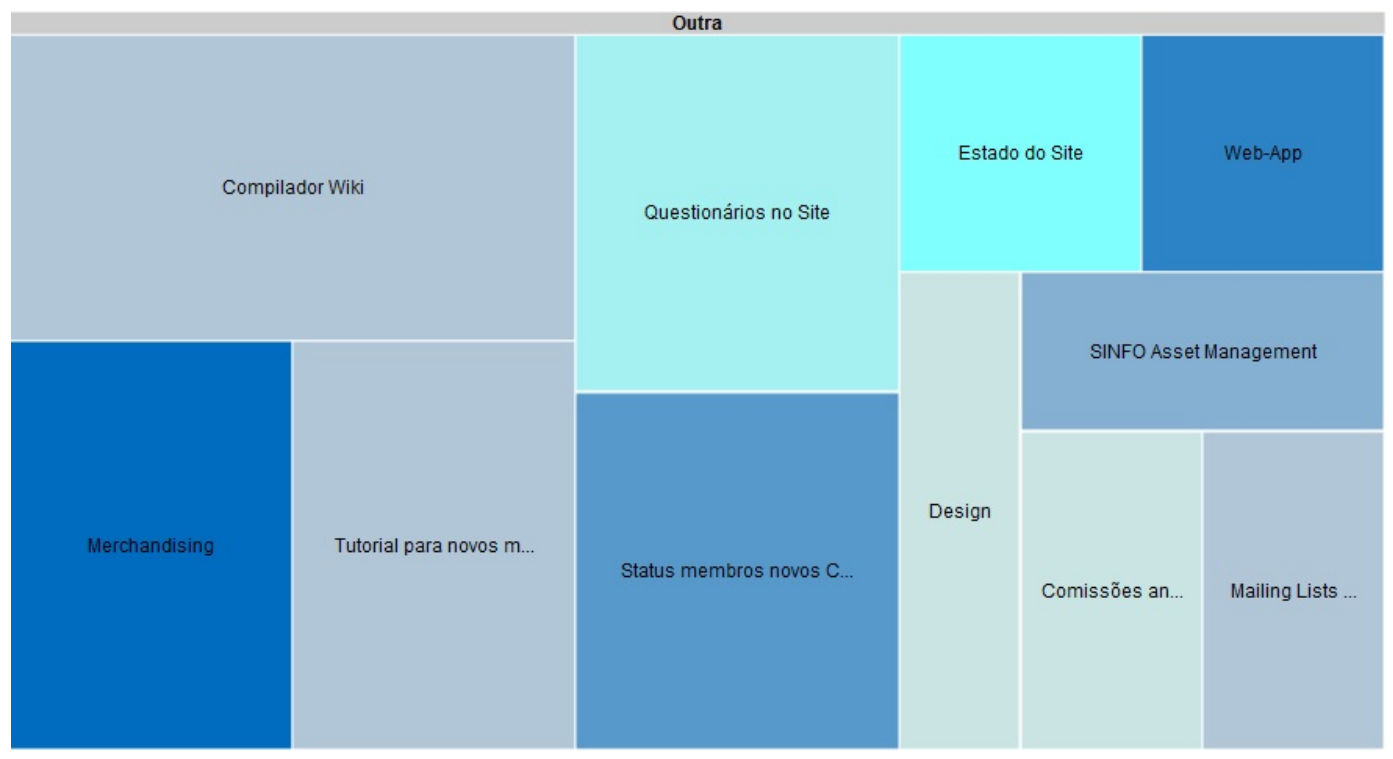

Fig. 1. Treemap for requirements visualization (2nd level)

\section{B. Visualization of Suggested Requirements}

Regarding the visualization referred to in III-A the prototype has a dashboard on its homepage with proposed bar charts to better inform the user of what has been going on in the elicitation activity. These charts show the most voted requirements, the users that submitted more requirements, the requirements that have more comments and the requirements that were more commented on in the last twenty-four hours. When a user sees the details of a requirement, a chart is also shown to illustrate the difference in votes between the most popular requirements and the one that is actually being seen.

The three alternatives for requirements visualization considered by our approach are implemented using the Google Charts API [48].

\section{Social Visualization}

The proposed social visualization based on a bubble chart is supported by the prototype using Fusion Charts [47]. Each stakeholder is represented by a bubble. The horizontal and vertical axis showed the number of comments and votes made by each stakeholder while the size of each bubble represents the sum of the suggested requirements, comments and votes (please see Figure 4).

The color of each bubble indicates the status that the stakeholder has achieved. Each contribution has equal value, and a different status is achieved after five contributions, meaning that to get to the bronze status and individual has to make more than five contributions, more ten to silver and more than fifteen contributions to achieve gold status. When the cursor is over a bubble, the username of its correspondent user is displayed. This visualization allows no customization following Erickson's guidelines [39] presented in II-C.

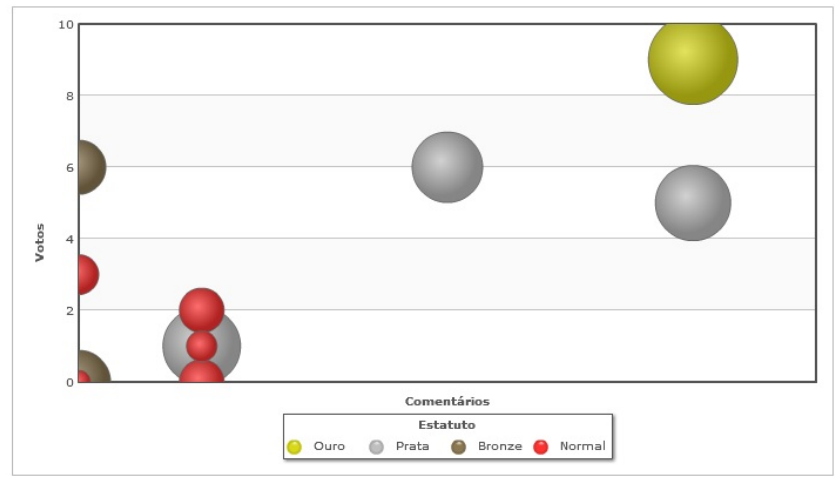

Fig. 4. Proposed social visualization

\section{Evaluation}

The prototype was evaluated according to "Goal-based evaluation of IT system as such" strategy [49]. This is a deductive approach that does not need user involvement and is used when it is intended to check if an IT artifact fulfills the intended goals.

This evaluation was performed by all the thirteen students of a requirements engineering subject from a Bsc in Management and Information Systems at the Higher Institute of Bank Management [50]. The prototype was demonstrated to these students in the final class of their course, afterwards a survey was answered by them.

The questionnaire consisted of two different parts, the first was based on four different questions, each one stating a goal of the system, and the respondents were asked to state if they agreed or not with each goal. The answer was based on a six point Likert scale, with 0 meaning "Totally disagree" and 5 "Totally agree".

The second part of the questionnaire asked the respondents to classify each of the proposed alternatives to visualize the 
suggested requirements. Evaluation of the proposed social visualization was also asked. This evaluation of the proposed visualizations was based on a six point Likert scale with 0 meaning "Don't Like" and 5 "Like".

The defined goals for the prototype are:

1) Involve more stakeholders in requirements elicitation, avoiding face-to-face meetings

2) Ease the understanding of the requirements through the different graphical visualizations

3) Encourage stakeholders to participate in requirements elicitation through the different graphical visualizations of requirements

4) Encourage stakeholders participate in requirements elicitation through the use of social visualization

The results of the questionnaire are described in the next two tables.

TABLE I

RESULTS FROM GOAL-BASED EVALUATION OF IT SYSTEM AS SUCH

\begin{tabular}{cccc}
\multicolumn{5}{c}{ Average Classification } \\
\hline Goal 1 & Goal 2 & Goal 3 & Goal 4 \\
\hline 4.15 & 4.08 & 3.61 & 3.54
\end{tabular}

TABLE II

RESULTS FROM PROPOSED ALTERNATIVES FOR REQUIREMENTS VISUALIZATION EVALUATION

\begin{tabular}{cccc}
\multicolumn{4}{c}{ Average Classification } \\
\hline Treemap & Motion Chart & 3D Tag Cloud & Textual List \\
\hline 4.00 & 4 & 3.85 & 3.31
\end{tabular}

The respondents to the questionnaire were also asked to rate the social visualization based on the bubble chart, which achieved an average score of 3.23 .

\section{EXPERIMENT}

According to action research methodology, our proposal has been evaluated through an experiment. The scenario was the elicitation of requirements for an information system that would facilitate the management and planning of an event, SINFO'2012 [51] at IST.

The stakeholders were invited to participate in requirements elicitation for this system through an e-mail sent to the event's mailing list, which had forty-three members. This list included the members of the team that organizes the event and members who were responsible for previous editions of the event. A total of fourteen stakeholders accepted to participate in elicitation and the activity lasted for twelve days.

Initially the categories to group requirements were defined with one of the leaders of the event's team and after that, the invitation e-mail was sent. E-mails to remind about this elicitation activity were sent at the fourth, eighth and twelfth (last) days of this experiment.

At the end of this experiment the participants were asked to respond to a questionnaire where they were asked about the frequency that they accessed the prototype and about some motivational factors to participate in elicitation. In the last part of the questionnaire, the respondents were asked to classify the different requirements visualizations that were offered.

\section{Results}

This section presents the results of the experiment described in the previous section. Figure 5 details the number of contributions per day through with a chart, invitation was sent at day and reminder e-mails were sent at day 4, 8 and 12 .

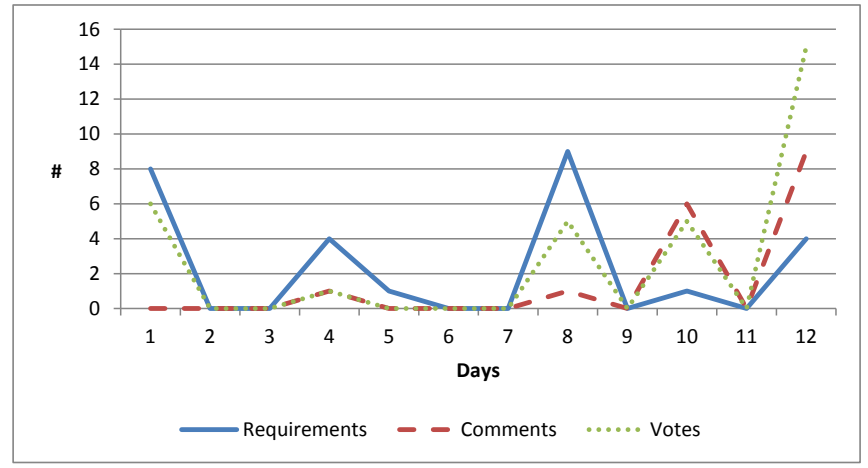

Fig. 5. Contributions per day

After the experiment, the participants were asked to answer a questionnaire that featured some motivational factors to participate in elicitation. Classification of the proposed requirements visualizations was also part of the questionnaire. Eleven of the fourteen individuals that participated in this experiment agreed to answer the questionnaire.

The questionnaire started by asking the frequency that the collaborative platform for elicitation was accessed by each user. This was a multiple choice question with possible answers being Once, More than once, but not daily, Daily, and Daily, more than once. Seven of the respondents accessed the platform more than once but not on a daily basis, three only accessed it once. Only one, accessed it daily, but this individual only decided to participate in the last day of the experiment.

The respondents were asked about the main reason to participate in elicitation, this was a multiple choice question that had the following possible answers options:

- Interest in project

- Liked that my opinion was asked

- Appeal from the project leaders

- Other

"Interest in project" was the answer with more choices, 6 . Following that, "Liked that my opinion was asked" had 3 choices and "Appeal from the project leaders" was chosen once. One respondent chose "Other" and specified "Future benefit for SINFO" as the main reason to participate.

The respondents were also asked if a game or the existence of rewards would increase their motivation to participate in elicitation. 5 of the 11 respondents answered that a game would motivate them to participate and 7 answered positively when asked if the existence of a reward would be a motivational factor.

The motivational impact of social visualization was also evaluated in this questionnaire with 8 of the 11 respondents stating that it motivates them to participate in elicitation. 
Table III presents the last part of the questionnaire's results, the classification of the proposed visualization by the experiment participants. The respondents were also asked to rate the proposed requirements visualizations using a six points Likert scale with 0 meaning "Don't Like" and 5 meaning "Like". In order to have a reliable rate, a log feature of the prototype was used, in order to check if a user really saw each of the visualizations that were rated.

TABLE III

CLASSIFICATION OF THE PROPOSED REQUIREMENTS VISUALIZATIONS

\begin{tabular}{c|cccc} 
Visualization & Max. rate & Min. rate & Average rate & Rated by \\
\hline Tabular List & 5 & 3 & 4.09 & 11 \\
Motion Chart & 5 & 0 & 2.89 & 9 \\
Treemap & 4 & 0 & 2.22 & 9 \\
3D Tag Cloud & 5 & 0 & 2.00 & 10
\end{tabular}

\section{EVALUATION}

The developed prototype enabled the different stakeholders to give their contributions and express their opinions independently of their location and without time constraints.

The majority of the stakeholders involved in this experiment considered social visualization as a motivational factor to participate in requirements elicitation.

According to the questionnaire's results the participants prefer the tabular list instead of the requirements visualizations that we propose. However, the proposed requirements visualization had some positive feedback, as can be seen by the higher rate of each one, but the rates from each user were very different which originated a lower average rate.

There is a need to frequently remind stakeholders to return to the elicitation platform, as it can be observed in Figure 5, since the main contributions related with new requirements were given in the days that a reminder e-mail was sent to the stakeholders. The questionnaire results also confirm that the users did not frequently access the elicitation platform. The number of comments and votes was higher in the last days of the experiment. Additionally there is a low rate of comments per requirement meaning that the discussion around requirements should be more stimulated. Analyzing the contributions from each user we can also find some different profiles:

- Users that mainly submit requirements (2)

- Users that submit few ideas but express their opinions through comments and votes (2)

- Users that only submitted votes (3)

- Users that did not contribute (3)

Despite the presence of a notification system based on comments, the cases where the author of a requirement continued the discussion around the commented idea were rare. This can either be interpreted as an absence of conflicts between stakeholders or that the comments made aimed at completing the description of each requirement.

According to our questionnaire the existence of rewards can boost the stakeholders' motivation to participate in elicitation. Associating a game to elicitation can also increase the motiva- tion of some of the stakeholders to contribute to requirements elicitation.

None of the requirements or comments were submitted anonymously. This shows that in this experiment none of the participants were worried with organizational barriers or felt intimidated to give their contribution.

\section{CONCLUSION}

This paper presents our proposal to collaboratively elicit requirements in a web-based environment. Complementary ways to visualize requirements are part of this proposal to stimulate user involvement and increase the perception of their relevance in the requirements elicitation activity. Social visualization is also proposed to engage stakeholders in this activity.

A prototype platform has been implemented and submitted to a goal-based evaluation. The results of that evaluation show that it accomplishes the proposed goals, which include the involvement of more stakeholders and better understanding of requirements. The requirements visualizations techniques also received positive feedback.

According to action research, our proposal has also been evaluated through an experiment that involved 27 requirements, 17 comments on the proposed requirements and 32 votes. The experimental results show that requirements elicitation can be made in an online collaborative environment. As a result, requirements elicitation can be supported in a quicker and cheaper way and can mitigate schedule or geographic constraints. The use of social visualization had a good impact on motivating users' participation. However the proposed requirements visualizations seem not to have the importance that we placed on them, since the participants in this experiment have expressed their preference for a traditional tabular view.

Regarding future work, we feel more experiments with different organizational and technical environments should be performed to obtain more results. Additional features to increase user engagement and the frequency of accesses to the online platform have to be considered. Namely techniques to design social interfaces like the addition of a personal dashboard, sharing or tagging features would be considered to this proposal.

\section{ACKNOWLEDGEMENT}

We would like to thank the participants in the presented experiment, especially the ones who kindly accepted to answer our questionnaire. We also thank the prototype evaluators.

\section{REFERENCES}

[1] K. Wiegers, Software Requirements, Second Edition. Microsoft Press, 2003.

[2] D. Zowghi and C. Coulin, "Requirements elicitation: A survey of techniques, approaches, and tools," Engineering and Managing Software Requirements, pp. 19-46, 2005.

[3] D. Damian, "Stakeholders in global requirements engineering: Lessons learned from practice," IEEE Software, vol. 24, no. 2, pp. 21-27, 2007.

[4] J. D. Herbsleb, "Global software engineering: The future of sociotechnical coordination," in 2007 Future of Software Engineering, ser. FOSE ’07, 2007. 
[5] H. Naz and M. N. Khokhar, "Critical requirements engineering issues and their solution," in Proceedings of the 2009 International Conference on Computer Modeling and Simulation. IEEE Computer Society, 2009.

[6] S. Kujala, M. Kauppinen, L. Lehtola, and T. Kojo, "The role of user involvement in requirements quality and project success," 13th IEEE International Conference on Requirements Engineering RE05, pp. 7584, 2005.

[7] T. S. Group, “Chaos summary 2009," 2009.

[8] S. Kujala, "User involvement: a review of the benefits and challenges," Behaviour Information Technology, vol. 22, no. 1, pp. 1-16, 2003.

[9] K. El Emam and N. H. Madhavji, "A field study of requirements engineering practices in information systems development," Decision Support Systems, vol. 14, no. 2, pp. 68-80, 1995.

[10] G. Beenen, K. Ling, X. Wang, K. Chang, D. Frankowski, P. Resnick, and R. E. Kraut, "Using social psychology to motivate contributions to online communities," in Proceedings of the 2004 ACM conference on Computer supported cooperative work, ser. CSCW '04. ACM, 2004.

[11] R. L. Baskerville, "Investigating information systems with action research," Commun. AIS, vol. 2, pp. 1-32, 1999.

[12] C. Farinha and M. Mira Silva, "Focus groups for eliciting requirements in information systems development," UK Academy for Information Systems Conference Proceedings, 2009.

[13] C. Farinha and M. Mira da Silva, "Web-based focus groups for requirements elicitation," in The Sixth International Conference on Software Engineering Advances, 2011.

[14] E. Davidson, "Joint application design (jad) in practice," Journal of Systems and Software, vol. 45, no. 3, pp. 215-223, 1999.

[15] B. W. Boehm and R. Ross, "Theory-w software project management principles and examples," IEEE Transactions on Software Engineering, vol. 15, no. 7, pp. 902-916, 1989.

[16] P. Grünbacher and B. Boehm, "Easywinwin: a groupware-supported methodology for requirements negotiation," SIGSOFT Softw. Eng. Notes, vol. 26, pp. 320-321, September 2001.

[17] M. Geisser and T. Hildenbrand, "A method for collaborative requirements elicitation and decision-supported requirements analysis," International Federation for Information Processing Digital Library, vol. 219, no. 1, 2006.

[18] V. Laporti, M. R. S. Borges, and V. P. Braganholo, "A collaborative approach to requirements elicitation," Proceedings of the 11th International Conference on Computer Supported Cooperative Work in Design, pp. 734-739, 2007.

[19] B. Decker, E. Ras, J. Rech, P. Jaubert, and M. Rieth, "Wiki-based stakeholder participation in requirements engineering," IEEE Software, vol. 24, no. 2, pp. 28-35, 2007.

[20] D. Ferreira and A. Rodrigues da Silva, "Wiki supported collaborative requirements engineering," in WikiSym '08: Proceedings of the 4th International Symposium on Wikis. ACM, 2008.

[21] A. Simha and R. Kishore, "Enhancing e-collaboration effectiveness through the use of wikis: A theoretical examination in the context of requirements elicitation." International Journal of e-Collaboration, vol. 5, no. 3, pp. 58-78, 2009.

[22] S. Lohmann and J. Ziegler, "Involving end users in distributed requirements engineering," Engineering Interactive Systems, pp. 221-228, 2008.

[23] C. Solis and N. Ali, "Distributed requirements elicitation using a spatial hypertext wiki," in Proceedings of the 2010 5th IEEE International Conference on Global Software Engineering. IEEE Computer Society, 2010.

[24] D. Yang, D. Wu, S. Koolmanojwong, A. W. Brown, and B. W. Boehm, "Wikiwinwin: A wiki based system for collaborative requirements negotiation," in Proceedings of the 41st Annual Hawaii International Conference on System Sciences HICSS 2008. IEEE Computer Society, 2008.

[25] E. Knauss, O. Brill, I. Kitzmann, and T. Flohr, "Smartwiki: Support for high-quality requirements engineering in a collaborative setting," 2009 ICSE Workshop on Wikis for Software Engineering, pp. 25-35, 2009.

[26] S. L. Lim, D. Damian, and A. Finkelstein, "Stakesource2 . 0 : Using social networks of stakeholders to identify and prioritise requirements," in Proceedings of the 33rd International Conference on Software Engineering. ACM, 2011.

[27] A. Rashid, D. Meder, J. Wiesenberger, and A. Behm, "Visual requirement specification in end-user participation," in Multimedia Requirements Engineering, 2006. MERE '06. First International Workshop on, sept. 2006.
[28] N. Seyff, F. Graf, and N. Maiden, "End-user requirements blogging with irequire," in Proceedings of the 32nd ACM/IEEE International Conference on Software Engineering - Volume 2, ser. ICSE '10. ACM, 2010.

[29] O. Gotel, F. Marchese, and S. Morris, "On requirements visualization," in Requirements Engineering Visualization, 2007. REV 2007. Second International Workshop on, 2007.

[30] J. Cooper, S.-W. Lee, R. Gandhi, and O. Gotel, "Requirements engineering visualization: A survey on the state-of-the-art," in Requirements Engineering Visualization (REV), 2009 Fourth International Workshop on, 2009.

[31] K. Pohl, Requirements Engineering. Springer, 2010

[32] (2012, Mar.) Agile Project Management, Requirements ,Management Gatherspace. [Online]. Available: http://gatherspace.com/

[33] (2012, Mar.) Requirements Managament Software. [Online]. Available: http://www.borland.com/us/products/caliber/index.aspx

[34] (2012, Mar.) IBM - Rational Requirements Composer - Software. [Online]. Available: http://www-01.ibm.com/software/awdtools/rrc/

[35] S. Lui, K. Lang, and S. Kwok, "Participation incentive mechanisms in peer-to-peer subscription systems," in System Sciences, 2002. HICSS. Proceedings of the 35th Annual Hawaii International Conference on, jan. 2002.

[36] P. J. Ludford, D. Cosley, D. Frankowski, and L. Terveen, "Think different: increasing online community participation using uniqueness and group dissimilarity," in Proceedings of the SIGCHI conference on Human factors in computing systems, ser. CHI '04. ACM, 2004.

[37] A. M. Rashid, K. Ling, R. D. Tassone, P. Resnick, R. Kraut, and J. Riedl, "Motivating participation by displaying the value of contribution," in Proceedings of the SIGCHI conference on Human Factors in computing systems, ser. CHI '06. ACM, 2006.

[38] J. Vassileva and L. Sun, "Using community visualization to stimulate participation in online communities," eService Journal, vol. 6, no. 1, pp. 3-39, 2007.

[39] T. Erickson, "Designing visualizations of social activity: six claims," in CHI 'O3 extended abstracts on Human factors in computing systems, ser. CHI EA '03. ACM, 2003.

[40] K. G. Karahalios and F. B. Viégas, "Social visualization: exploring text, audio, and video interaction," in CHI 'O6 extended abstracts on Human factors in computing systems. ACM, 2006.

[41] R. Cheng and J. Vassileva, "User motivation and persuasion strategy for peer-to-peer communities," in System Sciences, 2005. HICSS '05. Proceedings of the 38th Annual Hawaii International Conference on, 2005.

[42] E. Gilbert and K. Karahalios, "Using social visualization to motivate social production," IEEE Transactions on Multimedia, vol. 11, no. 3, pp. 413-421, 2009.

[43] C. Crumlish and E. Malone, Designing Social Interfaces. OReilly, 2009.

[44] B. Johnson and B. Shneiderman, "A space-filling approach to the visualization of hierarchical information structures," Information Visualization, pp. 284-291, 1991.

[45] M. A. Hearst and D. Rosner, "Tag clouds: Data analysis tool or social signaller?" Proceedings of the 41st Annual Hawaii International Conference on System Sciences HICSS 2008, no. June, pp. 160-160, 2008.

[46] (2012, Jan.) Outsystems: Agile development for enterprise web applications. [Online]. Available: http://www.outsystems.com/

[47] (2012, Jan.) Fusion charts - javascript (html5) \& flash charts. [Online]. Available: http://www.fusioncharts.com/

[48] (2012, Jan.) Google chart tools - google code. [Online]. Available: http://code.google.com/intl/en-en/apis/chart/

[49] S. Cronholm and G. Goldkuhl, "Strategies for information systems evaluation- six generic types," Journal of Information Systems, vol. 6, no. 2, pp. 65-74, 2003.

[50] (2012, Mar.) ISGB - The Portuguese School of Bank Management. [Online]. Available: http://www.isgb.pt/

[51] (2012, Mar.) Sinfo 2012. [Online]. Available: http://www.sinfo.ist.utl.pt/ 